\title{
Modelling of flow in pipes and ultrasonic flowmeter bodies
}

\author{
Richard Matas $^{1,}$, Vaclav Cibera ${ }^{1}$ and Tomas Syka ${ }^{1}$ \\ ${ }^{1}$ University of West Bohemia in Pilsen, NTC, Univerzitni 8, 30614 Plzen, Czech Republic
}

\begin{abstract}
The contribution gives a summary of the flow modelling in flow parts of ultrasonic flowmeters using CFD system ANSYS/FLUENT. The article describes the basic techniques used to create CFD models of flow parts flow and selected results of the flow fields. The first part of the article summarizes the results of velocity profiles in smooth pipes for various turbulent models and used relations. The second part describes selected results of the numerical modelling of flow in the flow parts of the ultrasonic flowmeters and their partially comparison with experimental results.
\end{abstract}

\section{Introduction}

Ultrasonic flowmeters are used more than fifty years and are applicable to liquids, gases and multiphase mixtures, but their applicability has limits [1]. This contribution describes selected results obtained during solution of research project concerning flow parts of ultrasonic flowmeter bodies for industrial applications.

The ultrasonic flowmeter mostly consists of the body and of the electronic control unit. The flow rate value is determined from the difference between the travel times of ultrasonic waves propagating in the measured liquid in and against the flow direction.

The work on the project was divided into several stages; selected of these are presented in the contribution. The first one is the solution of velocity profiles for the developed flow in the circular pipe. The next one was the modelling of flow in the flowmeter bodies without and with hydraulic perturbations. Very interesting was the modelling of flow in the body of the three-path ultrasonic flowmeter.

The goal of the work was to obtain of the flow field and velocity profiles for development of the flowmeter bodies and software algorithms for electronic control units. The project was also focused on influence of the hydraulic perturbations of liquids to the flow field and measured values. Numerical modelling and experimental research was performed during the project to obtain data about behaviour of flow in measuring instruments.

\section{Flow in parts of ultrasonic flowmeter}

Bodies of ultrasonic flowmeters can be designed as a shaped casting or a weldment, both design are finished by precision machining. The shape of the body should be simple for efficient manufacturing and to have low influence to the flow field. In some cases, the body includes inlet and outlet cone.

Ultrasonic probes are added to the main body. The figure 1 shows some examples of flowmeter bodies with pockets for probes.

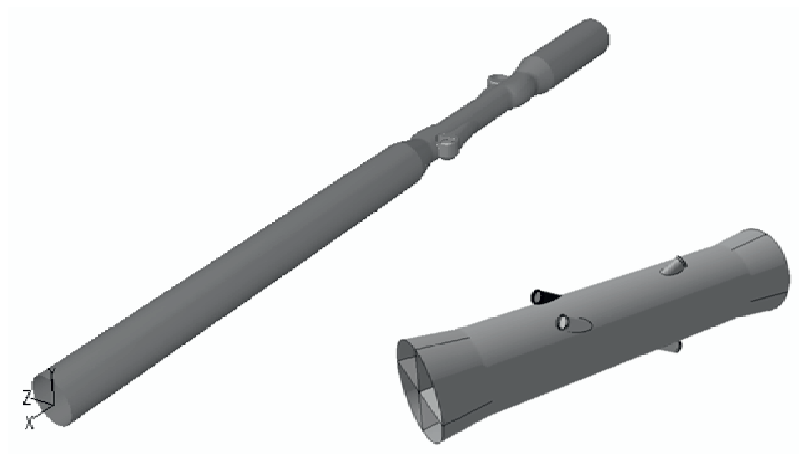

Figure 1. Examples of flowmeter bodies.

\subsection{Flow in pipe}

The flowmeters are mounted into pipes and the length of the straight section before should be sufficiently long to stabilize the flow. The pipe can be also supposed as a basic shape of the flowmeter body. Velocity profile is crucial for accuracy of the flowmeter. The one- or twopath flowmeters are calibrated in the testing laboratory and the calibration curve is entered into control unit. In case of multi-path flowmeters it is necessary to solve more complicated problem.

Multi-paths arrangement gives markedly wide information about flow and the flowmeter can achieve higher accuracy. The software integrates the values from probes of individual paths, various methods are used, and suitable method is, for example, OWICS [2]. The software in instrument can make corrections of flow-

\footnotetext{
${ }^{\mathrm{a}}$ Corresponding author: mata@ntc.zcu.cz
} 
rate with the help of values from single paths and perhaps even data from thermometer and viscosimeter. The computational procedures take into account Reynolds number, temperature and viscosity of the fluid and flow disturbances.

The basic turbulent velocity profile is the profile of developed flow without disturbances. Several methods were used to obtain this type of profile. The profiles were compared only in main flow, because the area close the wall wasn't important for that purpose.

\subsubsection{Analytical velocity profiles}

The formula for developed flow profile in smooth pipe can be found in various forms.

The first one is the power law profile. The formula for the velocity $u$ is simple and the relationship 1 can be written in the following form:

$$
\begin{gathered}
u=\bar{u} \cdot\left(n_{0}+1\right) \cdot\left[1-\left(\frac{r}{R}\right)^{2}\right]^{n_{0}} \\
\frac{1}{n_{0}}=1+\sqrt[6]{\frac{R e}{50}}
\end{gathered}
$$

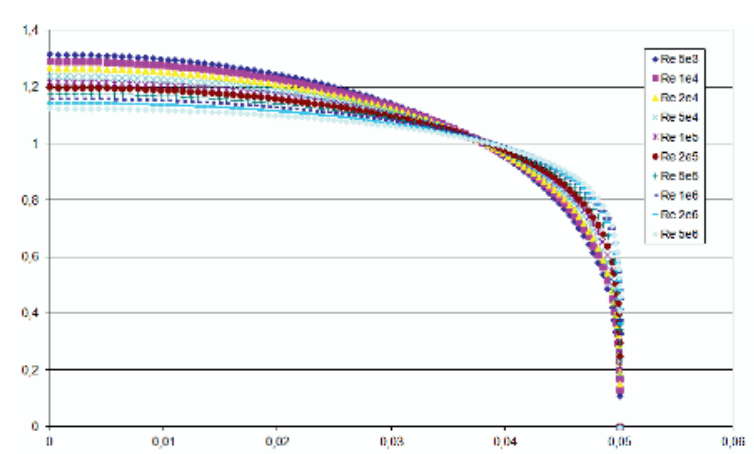

Figure 2. Velocity profiles - relationship 1.

More complicated power law are defined as:

$$
\begin{gathered}
u=u_{\max } \cdot\left[1-\left(\frac{r}{R}\right)\right]^{n_{0}} \\
\frac{1}{n_{0}}=1+\sqrt[6]{\frac{R e}{50}} \\
\frac{1}{n}=1.03 \ln (R e)-3.6 \\
m=\frac{u_{s}}{u_{\max }}=\frac{2}{(n+2) \cdot(n+1)}
\end{gathered}
$$

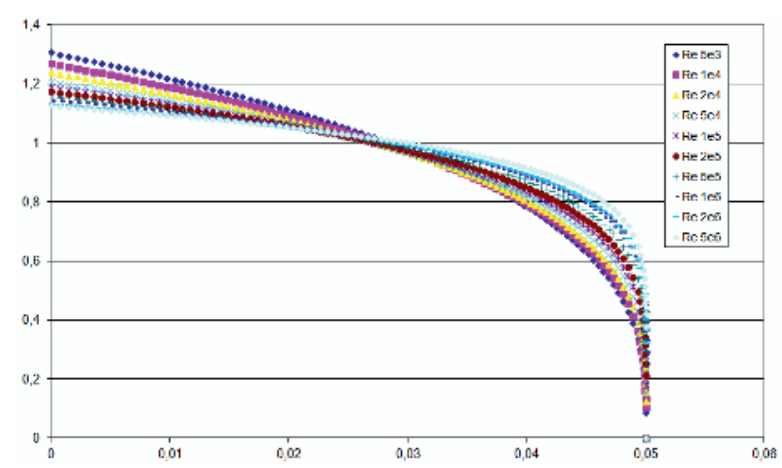

Figure 3. Velocity profiles - relationship 2.
Another form of the relationship is the logarithmic law of the profile:

$$
\begin{gathered}
u=\bar{u} \cdot\left[\beta u_{\text {lam }}+(1-\beta) \cdot u_{\text {turb }}\right] \\
u_{\text {lam }}=2 \cdot\left(1-\frac{r^{2}}{R^{2}}\right) \\
u_{\text {turb }}=1+\frac{2.652+1.768 \ln (1-r / R)}{3.48-1.74 \ln \left(41.7 / R e^{0.9}\right)} \\
\beta=\frac{1}{1+\left(R e / R e_{0}\right)^{4}} \\
\operatorname{Re}=\frac{2 R}{v} \cdot \bar{u}
\end{gathered}
$$

The profile should interpolate the experimental results and for the practical using seems usable.

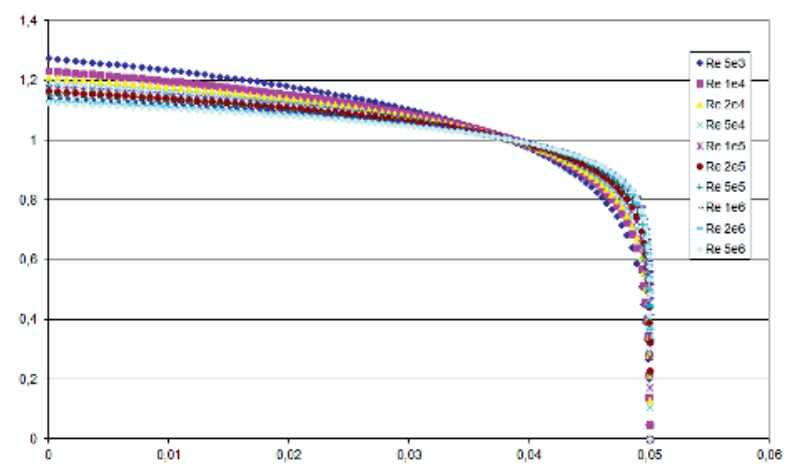

Figure 4. Velocity profiles - relationship 3.

Interesting solution of the analytical velocity profile is described in [3]. It regards the power law profile depending on the pressure gradient.

$$
\begin{gathered}
v=\bar{v} \cdot \frac{(N+3)}{(N+1)} \cdot\left[1-\left(\frac{r}{R}\right)^{N+1}\right] \\
N=\frac{R^{2}}{2 \mu \bar{v}} \cdot \frac{p_{1}-p_{2}}{L}-3
\end{gathered}
$$

The advantage of the relationship is the simplicity and wide use. The disadvantage is the need to know the pressure drop and inaccuracy in the published version for now, see Figure 5. The value for the pressure gradient in (13) was obtained from CFD computations because the experimental values weren't available. The profiles seem to be too flat, but the development of the relationship is still in progress.

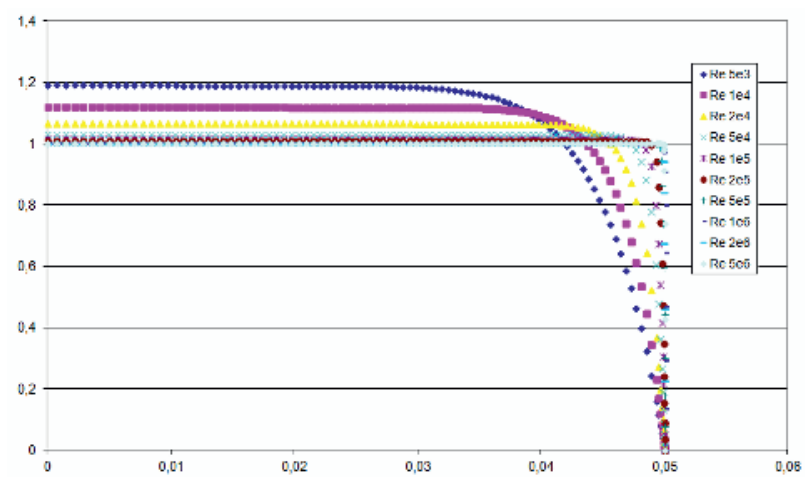

Figure 5. Velocity profiles - relationship 4. 


\subsubsection{Shape of the velocity profiles derived from the theory of large eddies}

Among the others mathematical models, it was investigated model found in [4]. Based on the NavierStokes equation and on the assumption that only large eddies plays crucial role for the final shape of velocity profile, there was in [4] derived new mathematical model to express the shape of velocity profile in pipes and between the plates. The most interesting fact for our work seemed to be that this model could be appropriately completed with easy measured data such as mean velocity of flow in pipes or among the plates. The solved equation for pipes is shown below (14).

$$
\begin{gathered}
C+v \frac{d^{2}\langle V\rangle}{d r^{2}}+v \frac{1}{r} \frac{d\langle V\rangle}{d r}=2 A\langle V\rangle \frac{d\langle V\rangle}{d r}- \\
3 B\langle V\rangle^{2} \frac{d\langle V\rangle}{d r}+\left(B V_{e f}^{2}-A V_{e f}\right) \frac{d\langle V\rangle}{d r}
\end{gathered}
$$

Where $\mathrm{A}, \mathrm{B}, \mathrm{C}$ are constant, $\mathrm{r}$ is pipe radius, $\mathrm{V}$ is velocity, $\mathrm{V}_{\mathrm{cl}}$ is velocity at the center of flow i.e. constant. A few of velocity profiles were computed according to methodology presented in [4]. But the problem arose about specific constants A, B in this model. The main reason for that was that these constants must be obtained based on "numerical experiments". Hence it was not sure, if the results were unambiguously. Anyway, this model approximated the shape of velocity profile well. So the conclusion of this experiment could be stated as follows: if there will be some methods derived out of the physical nature of the phenomena to acquire constants A and $\mathrm{B}$, the equation stated above has a great potential to describe the shape of the velocity profile sufficiently.

\subsubsection{Numerical computed velocity profiles}

Numerical methods are currently widely used for solution of developed turbulent flow fields. Applying of turbulent models can help to solve relatively complicated flow structures, but in this case it is necessary to solve one of basic problems in fluid dynamics - developed turbulent flow in the smooth tube. The work directed to the simple stationery axisymmetric model, see Figure 6.

The system ANSYS/FLUENT was used for simulations. The model for the solution was very simple; the rectangle computed domain was meshed with quad mesh, the boundary condition on the inlet and outlet was set as periodic with defined mass flow rate depending on Reynolds number ( $\mathrm{Re}$ ) and the fluid was defined as incompressible. The following RANS turbulent models were tested: k- $\varepsilon$, RNGk-e, SSTk- $\omega$ and RSM, for Re smaller than $2 \cdot 10^{4}$ were tested the modifications of models for low Reynolds numbers.

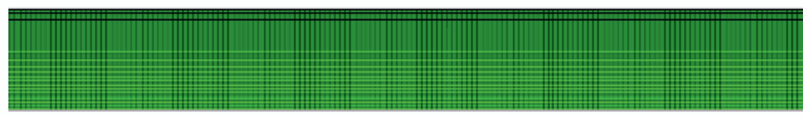

Figure 6. Axisymmetric model for CFD simulation.
In Figure 7 you can see the velocity profiles for RNGk-e model for various Re, which is widely used for engineering applications.

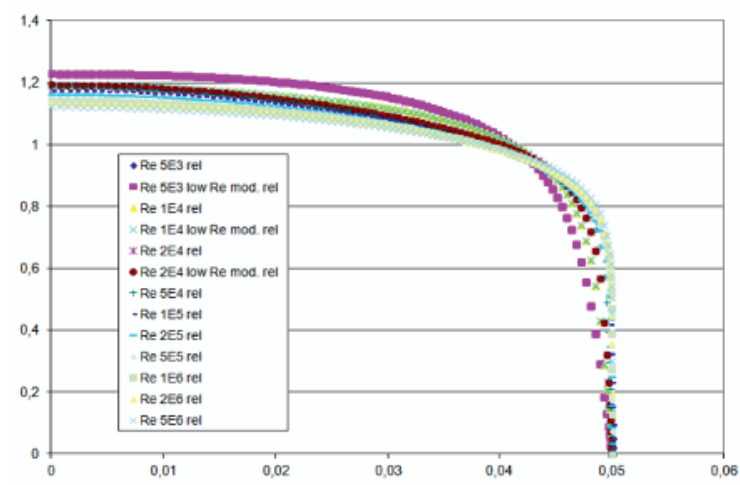

Figure 7. Velocity profiles - RNGk- $\varepsilon$ model.

In Figure 8 are depicted velocity profiles for other "engineering" model SSTk-w, which has become in the recent years very popular.

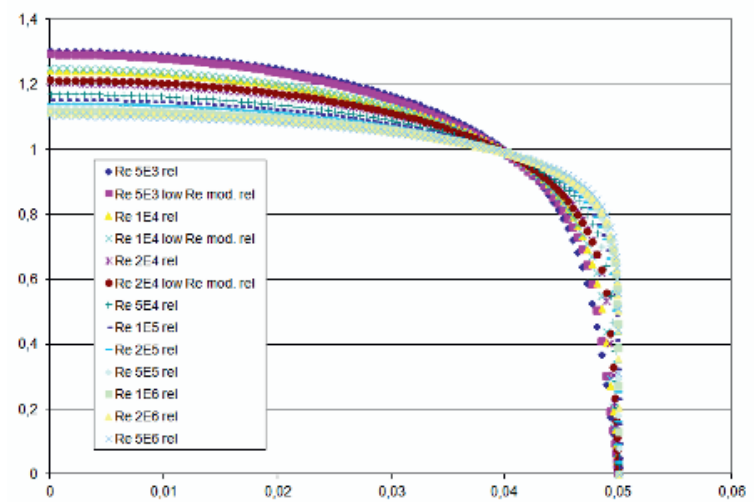

Figure 8. Velocity profiles - SSTk- $\omega$ model.

\subsubsection{Results}

To assess the results it is necessary to compare the results of all models. Four values of Re was selected to see all models in one diagram $-5 \cdot 10^{3}, 2 \cdot 10^{4}, 2 \cdot 10^{5}$ and $5 \cdot 10^{6}$. The Figures $9-12$ show the velocity profiles for all used relationships and models.

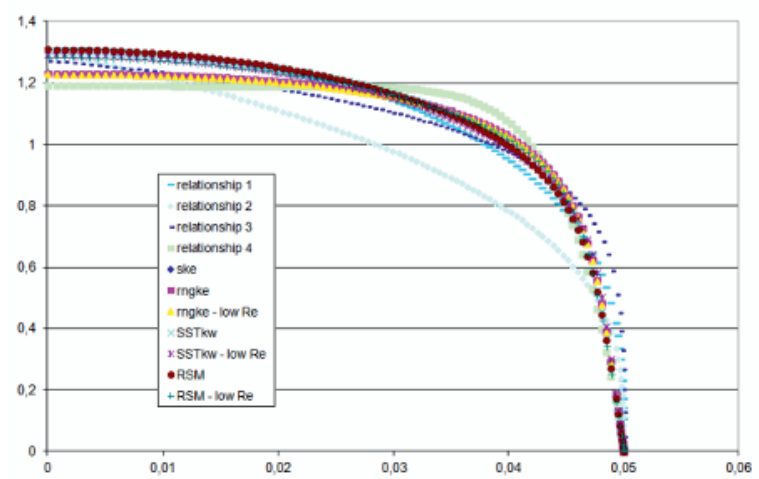

Figure 9. Velocity profiles $-\mathrm{Re}=5 \cdot 10^{3}$. 


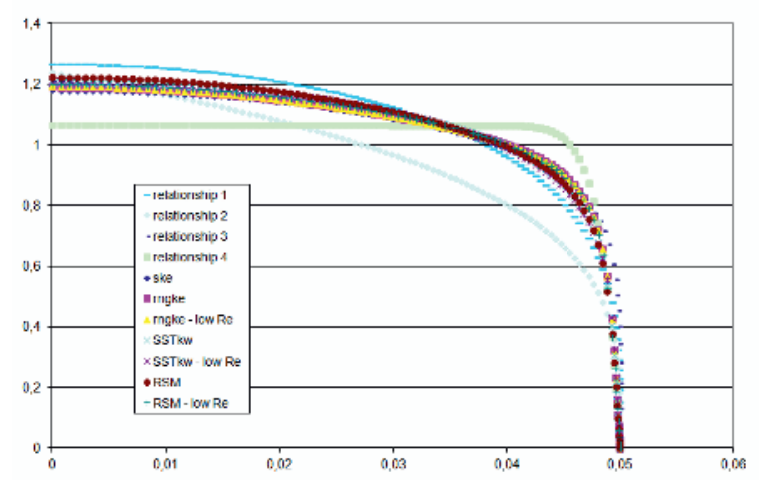

Figure 10. Velocity profiles $-\mathrm{Re}=2 \cdot 10^{4}$.

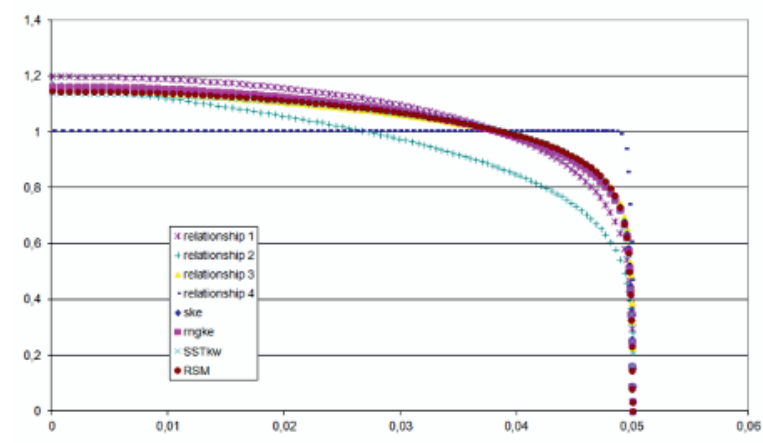

Figure 11. Velocity profiles $-\mathrm{Re}=2 \cdot 10^{5}$.

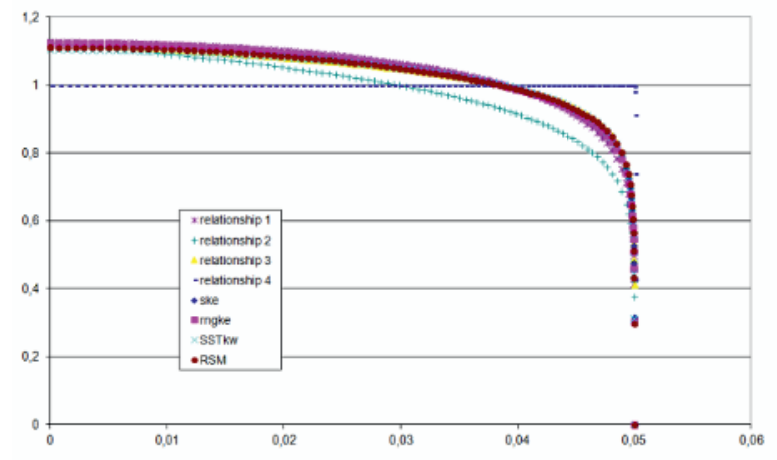

Figure 12. Velocity profiles $-\operatorname{Re}=5 \cdot 10^{6}$.

The graphs show different behaviour for used relationships and turbulent models. Values closest to the experimental results can be expected in relationship 3 , but for lower Re you can see the discontinuous derivative in the centre of the tube. From these results, it is seen disadvantages relations 1,2 (too sharp profiles) and 4 (too flat profile for published parameters). It can be stated, that the relationship 1 can be used as a simple and relatively exact for "engineering" application.

The simple numerical models show smaller differences than relationships, but even here the values differ. After evaluating the results, it seems that the most reliable results give the models SSTk- $\omega$ and RSM.

\subsection{Flow in ultrasonic flowmeter bodies}

The turbulent flow in bodies of ultrasonic flowmeters was simulated, similar simulations are performed $[5,6]$

The geometries were based on data from the industrial partner. The following paragraphs show one of the many variants that were simulated. The dimensions of the flowmeter bodies were from DN 50 to DN 200.

The computational meshes used for simulations were $3 \mathrm{D}$ tetrahedral or hybrid with the boundary layer. The number of cells was about 7 millions. The steady and unsteady flow of incompressible fluid was simulated, the boundary conditions were set depending on the required conditions.

The published results were obtained for the model flow meter DN 80 (2- paths). The flowmeter was equipped with a simple "crosswise" conditioner (see Figure 1 left). The flow was modelled as unsteady turbulent with mass flow inlet $21.4 \mathrm{~kg} / \mathrm{s}$. The pressurebased solver was used.

The RSM model should better describe the complex flow field, but the simulations can be in same cases unstable. The model SSTk- $\omega$ was preferred in simulations of the "real flowmeter geometries". In some cases were tested simulations by using of LES method.

In the Figure 13 you can see the flow field in the flowmeter. The flow field is in this design relatively smooth; it is possible to observe unsteady flow in the rear recess, where there are high levels of RMS values.

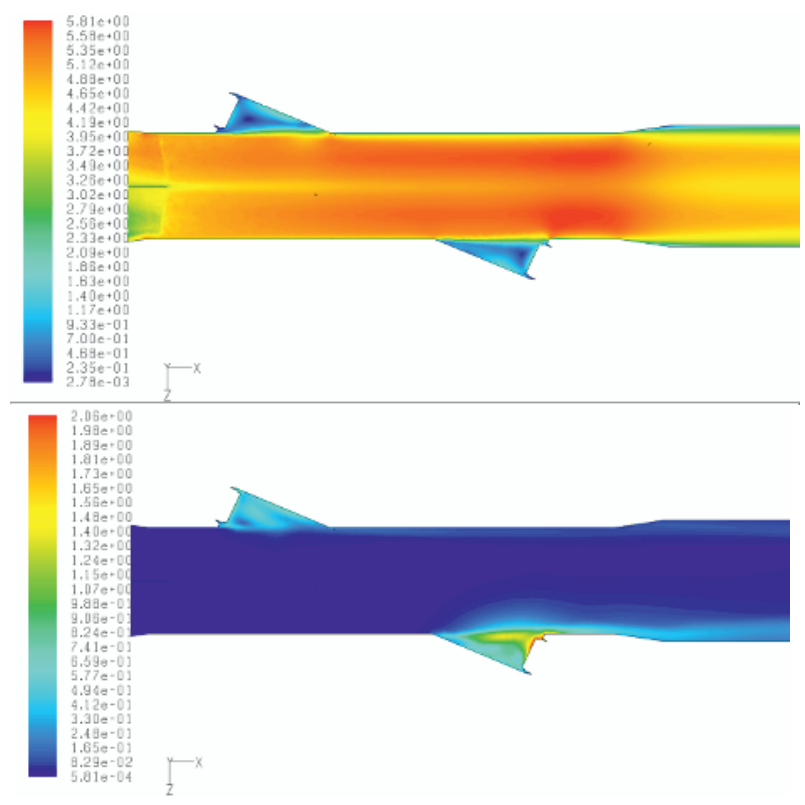

Figure 13. Contours of mean velocity magnitude and RMS velocity in the slice of the model of 2-path flowmeter.

Calculated results generally showed the need for minimizing the recesses for ultrasonic probes and the sensitivity of the flowmeter bodies to a number of other geometric parameters.

\subsection{Influence of the flow perturbations}

The flow perturbations can affect the accuracy of the flowmeter. They can be of different types and in practice produce a number of factors.

It turned out that for the measurements using the ultrasonic flowmeters is very dangerous the so called swirl perturbation, see Figure 14. The effect of this 
perturbation is again dependent on the conditions and in particular on the configuration of the body.

\subsubsection{Numerical model}

Numerical model of the swirl perturbation (and perturbations generally) was prepared as modular as to minimize the time required to prepare. The tetrahedral mesh was used and the model was added to the model of flowmeter body without perturbation. The length of pipe between the perturbation and flowmeter was 5D for 2-paths flowmeters, 10D for 1-path flowmeters and was tested also the configuration with short length 1.5D.

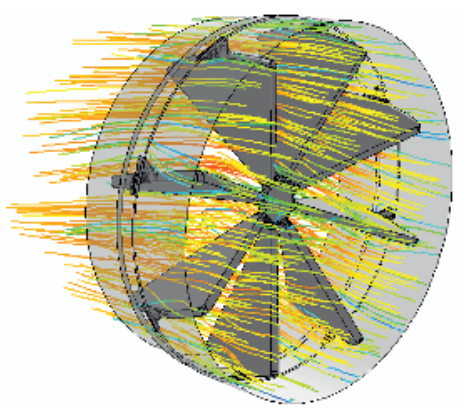

Figure 14. Numerical model of the swirl perturbation.

\subsubsection{Results}

In the Table 1 and in Figure 15 you can see the comparison of results with and without swirl perturbation for case from section 2.2 .

Table 1. Averaged velocities and their RMS values on the flow paths.

\begin{tabular}{|c|c|c|}
\hline & Velocity $(\mathrm{m} / \mathrm{s})$ & RMS Vel. (m / s) \\
\hline & \multicolumn{2}{|c|}{ Path 2} \\
\hline no perturbation & 3.6779 & 0.1190 \\
\hline rotat. perturbation & 3.6396 & 0.0677 \\
\hline & \multicolumn{2}{|c|}{ Path 2} \\
\hline no perturbation & 3.6598 & 0.0896 \\
\hline rotat. perturbation & 3.6115 & 0.1333 \\
\hline
\end{tabular}
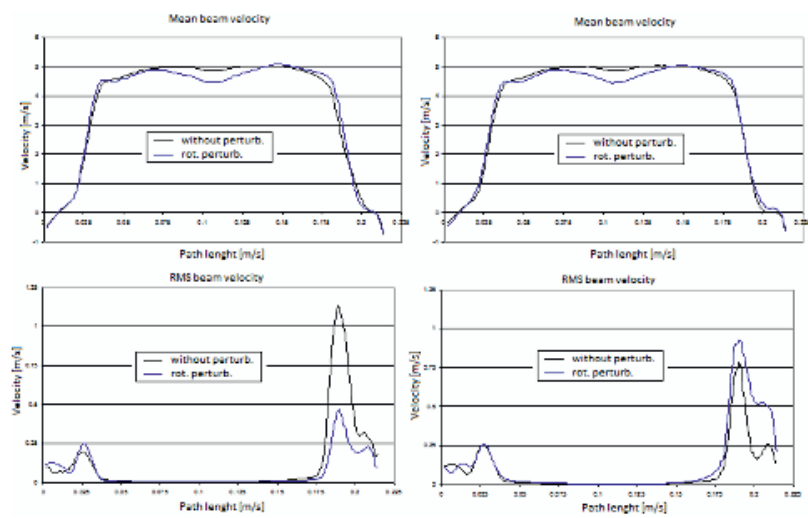

Figure 15. Distribution of velocity along flow paths for situation without and with the swirl perturbation.
It is possible to observe that the results with and without disturbances are relatively similar. It is a consequence of the flowmeter is equipped with a simple flow conditioner, the difference is roughly twice without this add-on.

\subsection{Model of the 3-path flowmeter body}

The 3-path flowmeter body has been developed taking into account a number of recommendations obtained by CFD and measurements on simpler flowmeter. Here are some results of dimension DN 100, which was the basic dimension for the project.

\subsubsection{Numerical model}

In Figure 16 you can see the entire modular model of the computational domain, including pipelines, flow conditioner, body flow and output section including dampers. This area was covered with computational mesh with the number of cells around 12 million. The setting of the model parameters was similar as in section 2.2. The model was solved with RANS SST k- $\omega\left(\mathrm{Re}=5 \cdot 10^{3}\right.$, $2 \cdot 10^{4}$ and $\left.5 \cdot 10^{5}\right)$ and LES method $\left(\operatorname{Re}=2 \cdot 10^{4}\right)$.

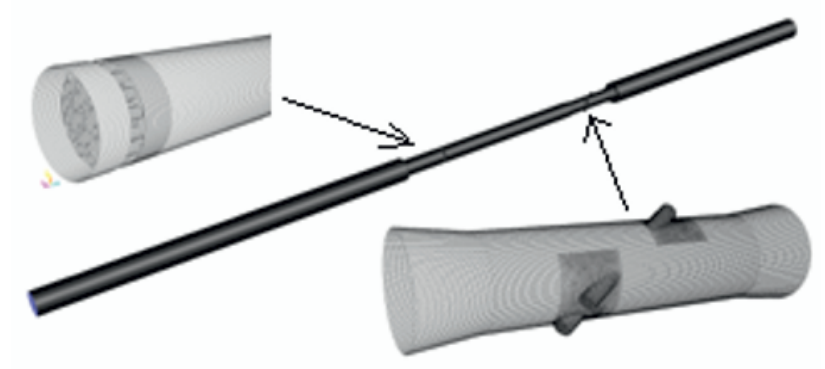

Figure 16. Geometry of the simulated area and surface mesh of the 3-path flowmeter body and flow conditioner.

\subsubsection{Results}

In the Figure 17 you can see the instantaneous unsteady flow structure in the duct.

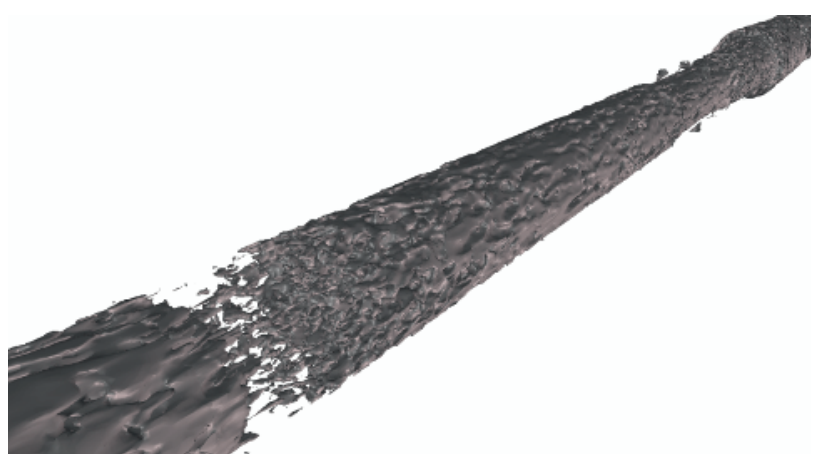

Figure 17. Vorticity surfaces in the channel, LES by $\operatorname{Re}=2 \cdot 10^{4}$. 

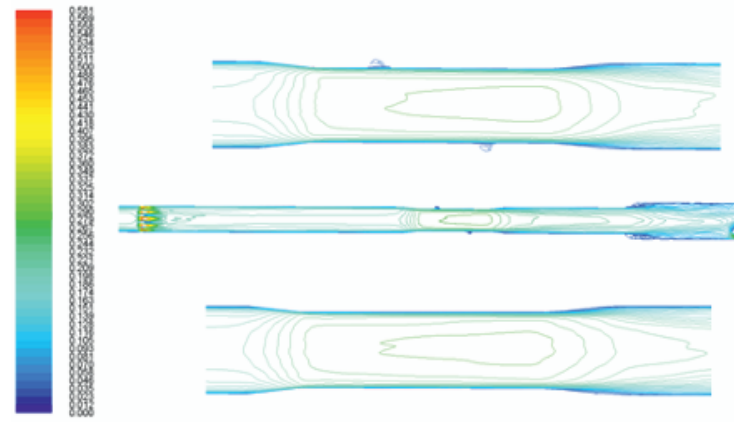

Figure 18. Computed flow field at $\operatorname{Re}=2 \cdot 10^{4}$.

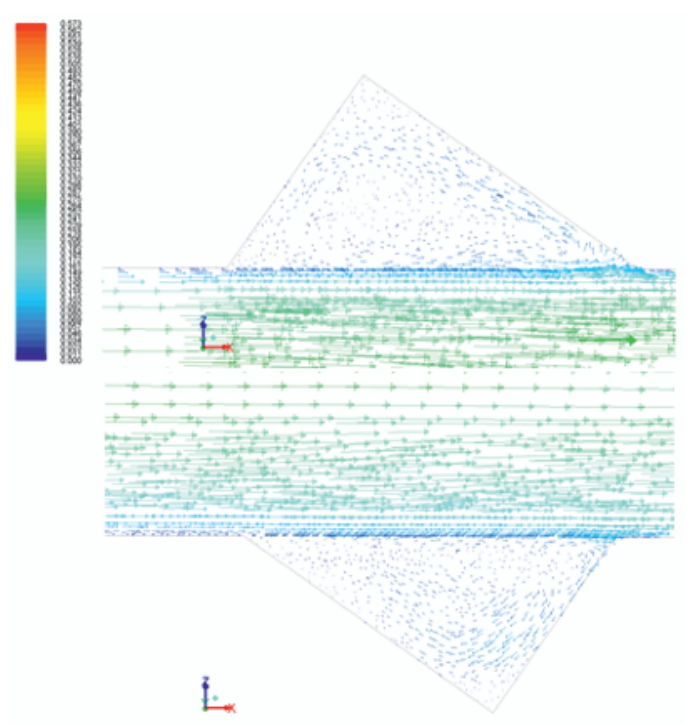

Figure 19. Velocity vectors in area of probes at $\operatorname{Re}=2 \cdot 10^{4}$.

In the Figure 18 are depicted the time averaged flow fields in the computational domain and in the flowmeter body. Figure 19 shows a relatively small effect of recesses for the probes on the flow field. Figure 20 and Table 2 show the difference between the model SST k- $\omega$ and LES. It is seen that the calculated velocities of flow on ultrasonic paths are virtually the same.

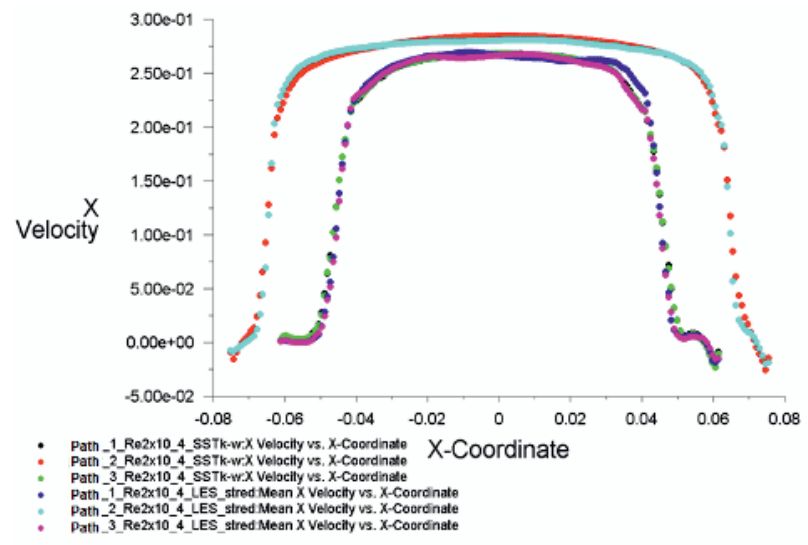

Figure 20. Axial velocities $(\mathrm{m} / \mathrm{s})$ on the flow paths for $\operatorname{Re}=2 \cdot 10^{4}$.
Table 2. Averaged velocities on the flow paths.

\begin{tabular}{|c|c|c|c|c|}
\hline model & $\operatorname{Re}$ & $\begin{array}{c}\text { Path 1 } \\
(\mathbf{m} / \mathbf{s})\end{array}$ & $\begin{array}{c}\text { Path 2 } \\
(\mathbf{m} / \mathbf{s})\end{array}$ & $\begin{array}{c}\text { Path 3 } \\
(\mathbf{m} / \mathbf{s})\end{array}$ \\
\hline SST k- $\omega$ & $5 \cdot 10^{3}$ & 0.046 & 0.059 & 0.046 \\
\hline SST k- $\omega$ & $2 \cdot 10^{4}$ & 0.189 & 0.230 & 0.189 \\
\hline $\begin{array}{c}\text { LES - instant. } \\
\text { value }\end{array}$ & $2 \cdot 10^{4}$ & 0.180 & 0.225 & 0.194 \\
\hline $\begin{array}{c}\text { LES - time } \\
\text { averaged value }\end{array}$ & $2 \cdot 10^{4}$ & 0.189 & 0.230 & 0.186 \\
\hline SST k- $\omega$ & $5 \cdot 10^{5}$ & 4.859 & 5.652 & 5.011 \\
\hline
\end{tabular}

\section{Conclusions}

It was solved and analyzed the flow in a smooth pipe and the bodies of the ultrasonic flowmeters. Based on the results it can be stated several findings.

The developed turbulent flow in the smooth pipe for various Reynolds numbers was solved as the first problem. The relationship 1 e.g. equations (1) and (2) was chosen as a suitable compromise describing the velocity profile. For the numerical simulations was chosen the two equation model SST k- $\omega$.

The simulation of ultrasonic flowmeter bodies has shown the flow field and its changes by influence of the hydraulic perturbations. The design modification has been also tested and optimised.

The model of the 3-path flowmeter body is very complex and calculation detailed maps flow behaviour under various conditions, and it is further continued.

It has been prepared the comparison between simulations and experimental testing of flow perturbations effects. The research should continue to bring as much information about the field described.

\section{References}

1. L.C. Lynnworth, Y. Liu, Ultrasonics 44, e1371 - e1378, (2006)

2. T. Tresch, P. Gruber, T. Staubli, Proceeding of the 6th International Conference on Innovation in Hydraulic Efficiency Measurements (2006)

3. J. Stigler, Proceeding of the 31th Meeting of Fluid Dynamics and Thermodynamics Departments, Mikulov, (2012)

4. A. E. Karpelson, arXiv:physics/9904030 (1999)

5. P. D. Lysak, D. M. Jenkins, D. E. Capone, W. L. Brown, Flow Measurement and Instrumentation, 19, Issue 1, 41-46 (2008)

6. H. Sheng, P. Lihui, H. Nakazato, Chinese Journal of Scientific Instrument, Vol. 30, No. 4 (2009)

\section{Acknowledgement}

These results were achieved in the framework of the FRTI1/126 project of the programme "TIP", Ministry of Industry and Trade of the Czech Republic programme and in specific research. 